\title{
Adolescent subdural empyema in setting of COVID-19 infection: illustrative case
}

\author{
Vladimir A. Ljubimov, MD, ${ }^{1}$ Robin Babadjouni, MD, ${ }^{1}$ Joseph Ha, BA, ${ }^{2}$ Viktoria O. Krutikova, MS, ${ }^{3}$ Jeffrey A. Koempel, MD, MBA, ${ }^{4}$ \\ Jason Chu, MD, MSc, ${ }^{2}$ and Peter A. Chiarelli, MD, $\mathrm{PhD}^{2}$ \\ ${ }^{1}$ Department of Neurosurgery, Cedars-Sinai Medical Center, Los Angeles, California; ${ }^{2}$ Division of Neurosurgery, Children's Hospital Los Angeles, Los Angeles, California; ${ }^{3}$ Touro \\ University College of Osteopathic Medicine, Henderson, Nevada; and ${ }^{4}$ Division of Otolaryngology, Children's Hospital Los Angeles, Los Angeles, California
}

\begin{abstract}
BACKGROUND Coronavirus disease 2019 (COVID-19) is an ongoing viral pandemic that has affected modern medical practice and can complicate known pathology. The novel severe acute respiratory syndrome coronavirus 2 (SARS-CoV-2) causes symptoms that may mimic a viral pneumonia, with potential for serious sequelae, including acute respiratory distress syndrome, coagulopathy, multiorgan dysfunction, systemic vascular abnormalities, and secondary infection.

OBSERVATIONS The authors describe a case of a 15 -year-old boy who presented with a right subdural empyema and sinusitis while having active COVID-19 infection. The patient initially presented with left-sided weakness, frontal sinusitis, and subdural empyema. Emergent surgery was performed for evacuation of empyema and sinus debridement. Samples of purulent material within the subdural space were tested for SARS-CoV-2 by reverse transcriptase polymerase chain reaction. The patient had a successful recovery and regained the use of his right side after combined treatment. To our knowledge, this is the first reported case of a bacterial subdural empyema associated with frontal sinusitis in a coinfected patient with COVID-19 without evidence of COVID-19 intracranial infection.
\end{abstract}

LESSONS A subdural empyema, which is a surgical emergency, was likely a superinfection caused by COVID-19. This, along with the coagulopathy caused by the virus, introduced unique challenges to the treatment of a known pathology.

https://thejns.org/doi/abs/10.3171/CASE21506

KEYWORDS case report; coagulopathy; COVID-19; craniotomy; intracranial subdural empyema; pediatric; SARS-CoV-2

Severe acute respiratory syndrome coronavirus 2 (SARS-CoV-2) is the coronavirus causing severe respiratory illness and responsible for the global coronavirus disease 2019 (COVID-19) pandemic. SARS-CoV-2 has significantly altered practice conditions of modern medicine, even in the setting of improved therapies and ongoing vaccination. $^{1}$ In addition to the severe pulmonary symptoms of COVID-19, numerous extrapulmonary manifestations include thrombosis, stroke, encephalopathy, anosmia, and ocular symptoms. ${ }^{2}$ These systemic effects are believed to arise from interaction of SARS-CoV-2 with the angiotensin-converting enzyme 2 (ACE2) receptor or other receptors for cellular entry found throughout the body. In the pediatric population, COVID-19 has an increased probability of mild symptoms, with a reported mortality rate of $0.09 \%{ }^{3}$
Cases do exist of children requiring advanced ventilatory support, including a case of severe respiratory disease requiring extracorporeal membrane oxygenation. ${ }^{3}$ In addition, children may also have cardiac dilation and multisystem inflammatory syndrome. ${ }^{3}$

"Intracranial subdural empyema" refers to pyogenic infection within the subdural space, with layered infectious debris characteristically adherent to the leptomeninges. It has high mortality if untreated by antibiotics and represents an emergent indication for surgical evacuation. ${ }^{4}$ Within the pediatric population, subdural empyema may originate from primary sites including the middle ear, the paranasal sinuses (most commonly the frontal sinus), and diffusely from an initial meningitis process. ${ }^{5}$ The spread of infection to the intracranial space may occur through thinned fibro-osseous structures, as well as via a hematogenous route.

ABBREVIATIONS ACE2 = angiotensin-converting enzyme 2; CNS = central nervous system; COVID-19 = coronavirus disease 2019; CT = computed tomography; FFP = fresh frozen plasma; INR = international normalized ratio; MRI = magnetic resonance imaging; PAPR = powered air-purifying respirator; $\mathrm{PCC}=$ prothrombin complex concentrate; PT = prothrombin time; RT-PCR = reverse transcriptase polymerase chain reaction; SARS-CoV-2 = severe acute respiratory syndrome coronavirus 2.

INCLUDE WHEN CITING Published January 24, 2022; DOI: 10.3171/CASE21506.

SUBMITTED September 30, 2021. ACCEPTED October 11, 2021.

(C) 2022 The authors, CC BY-NC-ND 4.0 (http://creativecommons.org/licenses/by-nc-nd/4.0/). 
Early presentation of subdural empyema may include fever, headache, and nausea, which can progress to hemiparesis, altered mental status, convulsion, and cranial nerve deficits. ${ }^{5}$

Here we present the first reported case of subdural empyema in the setting of active COVID-19 infection, and we discuss the interplay between the two pathological entities, as well as the effect of positivity for COVID-19 on treatment considerations. Even with a positive diagnosis of COVID-19 on nasal swab SARS-CoV-2 reverse transcriptase polymerase chain reaction (RT-PCR), intracranial purulent material remained negative for SARS-CoV-2.

\section{Illustrative Case}

\section{Presentation and History}

A 15-year-old boy presented as a transfer to our institution due to new onset of hemiplegia, and a computed tomography (CT) scan showed evidence of a 5-mm hemispheric subdural fluid collection without midline shift. The patient was previously healthy without any known medical problems. Before transfer, he experienced 1 week of fever and flulike symptoms, followed by 4 days of nausea/vomiting and rapidly progressive left-sided weakness. On arrival, the patient was febrile $\left(39^{\circ} \mathrm{C}\right)$ with moderate confusion, was able to follow commands at full strength on the right side, and had decreased sensation and no movement of the left hemibody/face. His preoperative laboratory test results showed he had an international normalized ratio (INR) of 2.0, white blood cell count $22(1000 / \mu \mathrm{L})$, Creactive protein $38.6 \mathrm{mg} / \mathrm{L}$ (normal reference lower than $1.0 \mathrm{mg} / \mathrm{L}$ ), ferritin $370 \mu \mathrm{g} / \mathrm{L}$, and lactate dehydrogenase 244 units/L. SARS-
CoV-2 positivity was confirmed by two independent nasal swab RTPCR tests on the day of admission, as well as serum antibody positivity (value 2.1 ; reference $<0.7$ ).

The patient was immediately taken for contrast-enhanced brain and cervical spine magnetic resonance imaging (MRI) and received transfusion with fresh frozen plasma (FFP) and vitamin $\mathrm{K}$ to correct coagulopathy. MRI confirmed a right-sided holohemispheric subdural empyema with edema of the underlying cortex. Combined evaluation of CT and MRI showed right fluid collection and inflammatory changes within the right frontal, maxillary, and ethmoid sinuses (Fig. 1), without obvious imaging evidence of bony dehiscence at the posterior table of the frontal sinus. There was no evidence of mastoiditis or air space involvement within the petrous temporal bone.

\section{Operative Procedure}

The patient was taken to the operating room with neurosurgery and otolaryngology teams, with strict adherence to the institutional COVID19 isolation protocol. Delivery of FFP reduced the patient's INR to 1.8; subsequent prothrombin complex concentrate (PCC) infusion improved his INR to 1.1. As coagulation values were being corrected, colleagues from otolaryngology performed bilateral nasal endoscopy with right maxillary antrostomy and direct right frontal sinus trephination. Irrigation and debridement of the sinuses were performed, yielding purulent debris in the right maxillary sinus and right frontal sinus. After sinus debridement and coagulopathy correction, the patient was repositioned for a right hemispheric craniotomy. Upon dural opening, copious purulent material was immediately encountered and was sent for laboratory evaluation, including SARS-CoV-2 RT-PCR and bacterial and fungal
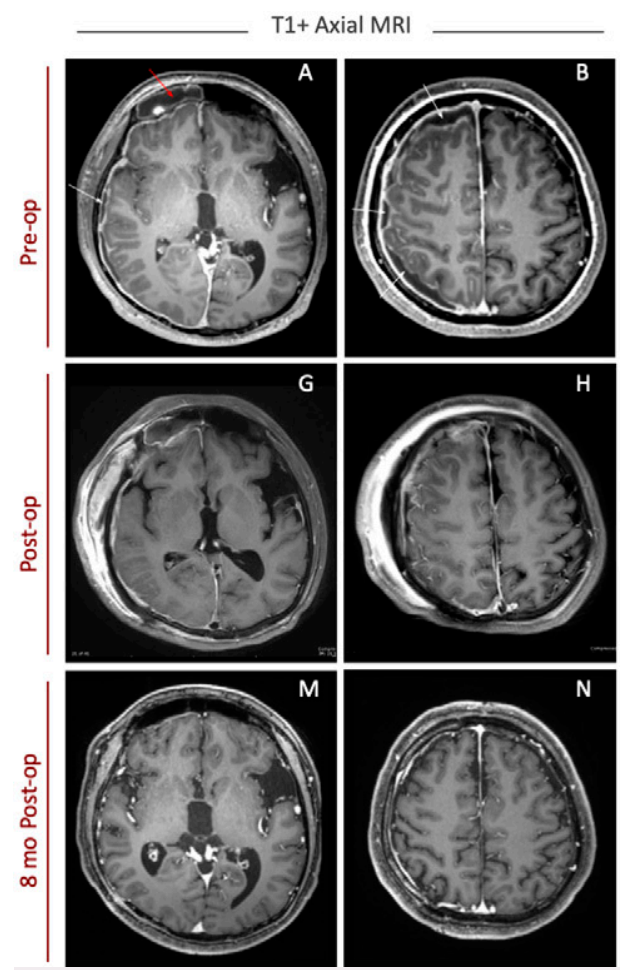

T2-W Axial
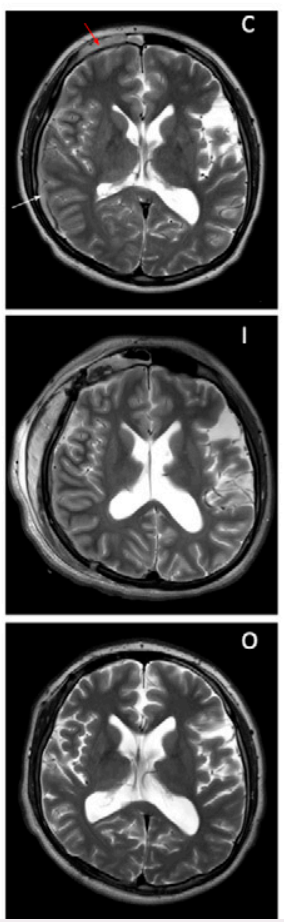

T1+ Corona
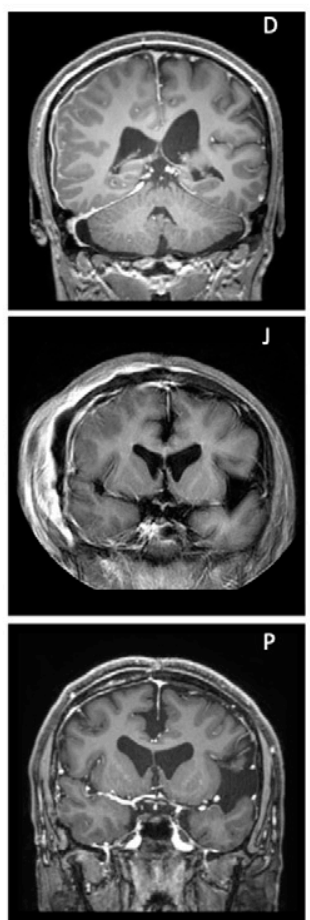

DW
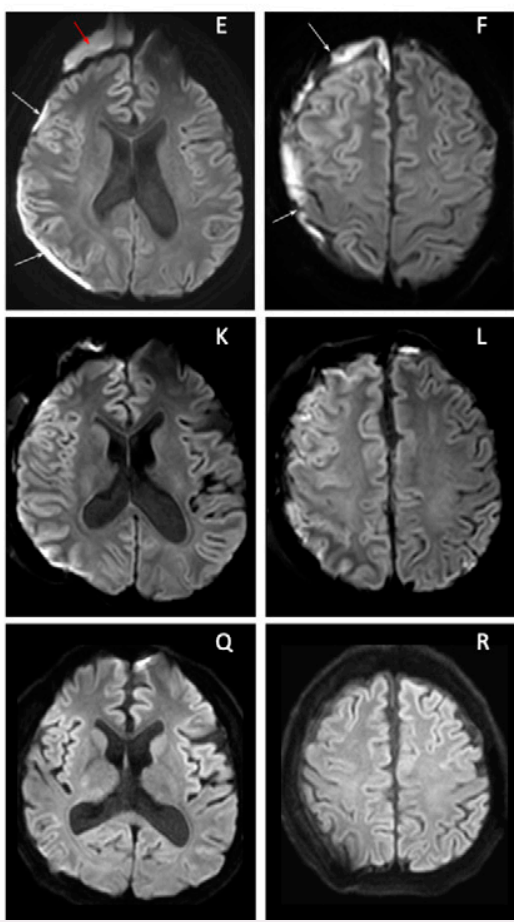

FIG. 1. MRI of the brain before surgery (A-F), immediately after surgery (G-L), and at 8-month follow-up appointment (M-R). White arrows point to imaging evidence of the subdural empyema, and red arrows show infected fluid collection in the right frontal sinus. Right-sided diffusion-restricted fluid collection, visible on presentation ( $E$ and $F)$, is substantially improved postoperatively $(K$ and $L)$ and is absent on follow-up imaging $(Q$ and $R)$. T1+ = T1weighted sequence with gadolinium contrast; $\mathrm{T} 2-\mathrm{W}=\mathrm{T} 2$-weighted sequence; $\mathrm{DWI}=$ diffusion-weighted imaging sequence. 
cultures. The surface of the brain and interhemispheric fissure were then irrigated with antibiotics and explored, clearing all evidence of liquid purulent material. Because the brain appeared significantly relaxed, the dura was reapproximated, and the bone flap was replaced with subgaleal drain placement. Postoperatively, the patient was transferred to the intensive care unit, remaining intubated.

\section{Postoperative Course}

The patient was extubated on the first postoperative day and was able to briskly follow commands on the right side. He regained trace movement of his left ankle shortly after extubation. He was started on broad-spectrum antibiotics and placed in COVID-19 isolation. Postoperative MRI showed successful subdural empyema evacuation without evidence of cortical restricted diffusion. Subdural and sinus cultures grew Streptococcus intermedius, and, in collaboration with our infectious disease team, we narrowed antibiotics to penicillin $G$ and metronidazole. The finding of SARS-CoV-2 RT-PCR of two independent intraoperative swabs of the subdural empyema in situ was negative.

Over the next 2 weeks, the patient continued to make improvements in motor strength and sensation. With intensive physical therapy, he regained complete strength of his left side and was able to walk and use his left arm functionally. The finding of follow-up nasal swabs for SARS-CoV-2 RT-PCR remained positive until 32 days after surgery, at which time the RT-PCR result was negative. The result of SARS-CoV-2 immunoglobulin $G$ antibody testing remained positive at the most recent test 2 months after surgery. MRI of the brain at 6 months after surgery showed no evidence of recurrent infection intracranially or in the sinuses.

\section{Discussion}

\section{Observations}

COVID-19 has numerous neurological manifestations, including viral encephalitis, acute cerebrovascular disease, and toxic encephalopathy. ${ }^{6}$ SARS-CoV-2 genetic material and proteins have been documented in the central nervous system (CNS) of affected individuals. ${ }^{6}$ The virus is thought to invade the CNS via blood circulation and retrograde transport through the olfactory nerves, which may lead to hypoxic injury; increased vascular permeability (i.e., loss of blood-brain barrier integrity); and autoimmune inflammatory changes, including changes of the capillary endothelium mediated directly via the ACE2 receptor. ${ }^{6}$

To our knowledge, COVID-19-associated intracranial subdural empyema has not been reported in the literature. Because the empyema itself was negative by SARS-CoV-2 RT-PCR, we hypothesize that the patient's COVID-19 upper respiratory infection created a favorable environment for bacterial sinusitis coinfection, subsequent intracranial extension, and subdural empyema formation. Our ear, nose, and throat colleagues reported that during surgery, the adenoid tissue obstructed bilateral choana by approximately $80 \%$ over normal, likely facilitating the development of sinusitis. Our novel finding was a negative SARS-CoV-2 RT-PCR result from the intracranial purulent material. This suggests that SARS-CoV-2 viral particles were not transmitted into the subdural space despite documented COVID-19 infection within the nares. Al-Olama et al. recently published a report on a patient with COVID-19 meningoencephalitis with resultant intraparenchymal hemorrhage and subdural hematoma. ${ }^{7}$ The subdural hematoma required burr hole evacuation in a delayed fashion, and the authors reported a positive RNA PCR result for
SARS-CoV-2 from the chronic subdural hematoma fluid. The authors did not speculate on the mechanism of transmission.

The appearance of secondary infection in the setting of SARSCoV-2 is now well described, ${ }^{8,9}$ whereas limited information remains available on the rate of COVID-19-related superinfection in the pediatric population. Xia et al. reported that the rate of pediatric secondary infection may be as high as $40 \%$, even though respiratory symptoms of both the primary and secondary infectious processes can be mild. ${ }^{8}$ A large metanalysis involving adult and pediatric patients noted a rate of COVID-19-related bacterial coinfection on presentation to be $3.5 \% .^{9}$ The rate of nosocomial superinfections in patients with COVID-19 was reported in the same study to be as high as $14.5 \%{ }^{9}$ In contrast, the rate of bacterial coinfections in patients with severe influenza was reported to be as high as $20 \%-30 \%$ across the literature. ${ }^{9,10}$ Musuuza et al. published a meta-analysis looking at hospitalized and nonhospitalized patients with COVID-19 with reported other infections and showed that bacterial coinfection/superinfection was seen in 4\%/6\%, respectively. ${ }^{10}$ The most common bacteria isolated in coinfected patients were Haemophilus influenzae, Mycoplasma pneumoniae, Streptococcus pneumoniae, Klebsiella pneumoniae, and Pseudomonas aeruginosa. ${ }^{9,10}$ The respiratory tract was the most common reported source of infection. ${ }^{9}$

Rhinosinusitis has been described in patients with COVID-19. One case series included two pediatric cases of complicated sinusitis in patients with COVID-19. ${ }^{11}$ In these cases, the sinusitis was associated with orbital cellulitis, orbital vein thrombosis, and meningeal enhancement and small intracranial epidural fluid collection behind the frontal sinus in one case. Both patients were treated with sinus washout and broad-spectrum antibiotics without intracranial surgery. In this report, no clear pathogen was identified. ${ }^{11}$ The authors hypothesized that compromised mucociliary clearance may have resulted in secondary sinus obstruction and thus bacterial infection. ${ }^{11}$ Another case series recounted two pediatric patients with COVID-19 complicated by rhinosinusitis. One patient with orbital cellulitis had an endoscopic right maxillary antrostomy, total ethmoidectomy, and frontal sinusotomy. Intraoperative cultures grew coagulase-negative Staphylococcus. ${ }^{12}$ The other patient presented with right-sided rhinosinusitis and orbital subperiosteal abscess. He was treated with broad-spectrum antibiotics and right orbitotomy and drainage of the abscess. Intraoperative cultures grew no organisms. ${ }^{12}$ Although the pathophysiology is still not entirely clear, these reports showcase how the bacterial superinfection causing rhinosinusitis may occur due to COVID-19.

In the present case, the patient presented with an elevated INR requiring correction. Although a septic or profound systemic inflammatory process may have contributed to coagulopathy, the described patient's blood culture results all remained negative, including those drawn before the initiation of antibiotics. ${ }^{13}$ COVID-19 has a documented association with coagulopathy and disseminated intravascular coagulation in acutely ill adult patients. ${ }^{2}$ Despite significant elevation in fibrin degradation product and D-dimer levels, only mild prothrombin time (PT) elevation to 13.6 seconds (INR 1.1-1.2), on average, has been observed in the hospitalized adult population with COVID-19. ${ }^{14}$ In subanalysis, the severely ill patients with COVID-19 who did not survive had the highest PT values of 15.5 seconds (INR 1.3-1.4), on average. ${ }^{14}$ However, Mitchell et al. reported 27 pediatric cases ranging in age from 2 months to 20 years with average PT increases to 17.8 seconds (INR 1.4-1.5), with the longest being 43.3 
seconds (INR 3.4-3.5), which may indicate a more pronounced response in the pediatric population. ${ }^{15}$ Potential contributing factors to the coagulopathy observed for our patient include systemic inflammatory changes associated with COVID-19 and inflammatory changes associated with sinusitis/empyema.

Documented hematological sequelae of COVID-19 also include thrombosis/hypercoagulability, ${ }^{16}$ which is relevant to the uncertain risk of multiple procoagulant blood product infusion in this case. Because immediate cranial surgery was needed and FFP/vitamin $\mathrm{K}$ failed to yield sufficient reduction of INR, PCC was required to achieve an INR $<1.6$ and thus minimize the potential for excessive intraoperative bleeding. Postoperatively, the patient underwent additional coagulation testing and had an elevated D-dimer of $850 \mathrm{ng} / \mathrm{mL}$ and fibrinogen of $1094 \mathrm{mg} / \mathrm{mL}$. No evidence of intravascular thrombosis was observed either on preoperative intracranial imaging or clinically throughout the patient's course of treatment. Further research is needed on how to address such issues to optimize preoperative care for neurosurgical patients, especially in an emergent setting.

During the patient's surgery, strict COVID-19 precautions were maintained. The neurosurgery team was using powered air-purifying respirator (PAPR) masks. The otolaryngology team used N-95 masks with plastic face shields for direct visualization through the sinus endoscope, which was not possible with the PAPR masks. A no-entry policy was maintained during the surgery to reduce operating room traffic and minimize the number of staff in close proximity with the patient. There was no transmission of the virus to the operative staff, which the authors attribute to the above effective safety measures.

\section{Lessons}

Subdural empyema represents a surgical emergency, and additional challenges are supplied to the acute management of this pathology by the presence of COVID-19. The cause of the empyema was also likely a secondary infection due to the initial viral illness. The patient's coagulopathy was also likely caused by COVID-19, and this needs to be evaluated acutely in pediatric surgical patients before surgery. Finally, a multidisciplinary team approach between primary and consulting teams is critical in such a complicated case.

\section{References}

1. World Health Organization (WHO). Coronavirus disease (Covid2019) situation reports. Accessed December 29, 2021. https://www. who.int/emergencies/diseases/novel-coronavirus-2019/ situation-reports.

2. Gupta A, Madhavan MV, Sehgal K, et al. Extrapulmonary manifestations of COVID-19. Nat Med. 2020;26(7):1017-1032.

3. Hoang A, Chorath K, Moreira A, et al. COVID-19 in 7780 pediatric patients: a systematic review. EClinicalMedicine. 2020;24:100433.

4. French H, Schaefer N, Keijzers G, Barison D, Olson S. Intracranial subdural empyema: a 10-year case series. Ochsner J. 2014;14(2): 188-194.

5. Konar S, Gohil D, Shukla D, et al. Predictors of outcome of subdural empyema in children. Neurosurg Focus. 2019;47(2):E17.
6. Wu Y, Xu X, Chen Z, et al. Nervous system involvement after infection with COVID-19 and other coronaviruses. Brain Behav Immun. 2020;87:18-22.

7. Al-Olama M, Rashid A, Garozzo D. COVID-19-associated meningoencephalitis complicated with intracranial hemorrhage: a case report. Acta Neurochir (Wien). 2020;162(7):1495-1499.

8. Xia W, Shao J, Guo Y, Peng X, Li Z, Hu D. Clinical and CT features in pediatric patients with COVID-19 infection: different points from adults. Pediatr Pulmonol. 2020;55(5):1169-1174.

9. Langford BJ, So M, Raybardhan S, et al. Bacterial co-infection and secondary infection in patients with COVID-19: a living rapid review and meta-analysis. Clin Microbiol Infect. 2020;26(12):1622-1629.

10. Musuuza JS, Watson L, Parmasad V, Putman-Buehler N, Christensen L, Safdar N. Prevalence and outcomes of co-infection and super-infection with SARS-CoV-2 and other pathogens: a systematic review and meta-analysis. PLoS One. 2021;16(5):e0251170.

11. Turbin RE, Wawrzusin PJ, Sakla NM, et al. Orbital cellulitis, sinusitis and intracranial abnormalities in two adolescents with COVID-19. Orbit. 2020;39(4):305-310.

12. Blanco $\mathrm{CH}$, Stein JB, Barinsky $\mathrm{GL}$, et al. Management of complicated pediatric rhinosinusitis in the COVID-19 era. Am J Otolaryngol. 2020;41(6):102746.

13. Walborn A, Williams M, Fareed J, Hoppensteadt D. International normalized ratio relevance to the observed coagulation abnormalities in warfarin treatment and disseminated intravascular coagulation. Clin Appl Thromb Hemost. 2018;24(7):1033-1041.

14. Tang N, Li D, Wang X, Sun Z. Abnormal coagulation parameters are associated with poor prognosis in patients with novel coronavirus pneumonia. J Thromb Haemost. 2020;18(4):844-847.

15. Mitchell WB, Davila J, Keenan J, et al. Children and young adults hospitalized for severe COVID-19 exhibit thrombotic coagulopathy. Pediatr Blood Cancer. 2021;68(7):e28975.

16. Hunt $B$, Retter A, McClintock $C$. Practical guidance for the prevention of thrombosis and management of coagulopathy and disseminated intravascular coagulation of patients infected with COVID-19. British Society for Haematology. Published online March 25, 2020. https://b-s-h.org.uk/media/18171/th-and-covid-25-march-2020final.pdf.

\section{Disclosures}

The authors report no conflict of interest concerning the materials or methods used in this study or the findings specified in this paper.

\section{Author Contributions}

Conception and design: Chiarelli, Ljubimov, Koempel. Acquisition of data: Chiarelli, Ljubimov, Krutikova, Koempel. Analysis and interpretation of data: Chiarelli, Ljubimov, Ha. Drafting the article: Ljubimov, Babadjouni, Krutikova, Koempel. Critically revising the article: all authors. Reviewed submitted version of manuscript: Chiarelli, Ljubimov, Babadjouni, Ha, Krutikova, Chu. Approved the final version of the manuscript on behalf of all authors: Chiarelli. Administrative/ technical/material support: Ha. Study supervision: Chiarelli.

\section{Correspondence}

Peter A. Chiarelli: Children's Hospital Los Angeles, Los Angeles, CA. pchiarelli@chla.usc.edu. 